\title{
APLICACIÓN Y COMPARACIÓN DE MÉTODOS UNIVARIADOS PARA EVALUAR LA ESTABILIDAD EN MAÍCES DEL VALLE TOLUCA- ATLACOMULCO, MÉXICO
}

\author{
Andrés González ${ }^{1 / *}$, Delfina de Jesús Pérez, Jaime Sahagún**, Omar Franco*, Edgar Jesús \\ Morales*, Martín Rubi*, Francisco Gutiérrez, Artemio Balbuena*
}

Palabras clave: Zea mays L., índices de estabilidad univariados, análisis de componentes principales. Keywords: Zea mays L., univariate stability indexes, principal components analysis.

Recibido: $20 / 03 / 10$

\section{RESUMEN}

El objetivo de este estudio fue aplicar 6 métodos univariados para evaluar la estabilidad de 25 genotipos de maíz (Zea mays L.) de los Valles Altos de México. Los genotipos fueron evaluados en 4 ambientes bajo un diseño de bloques completos al azar, con 4 repeticiones por ambiente. Con los datos del rendimiento de grano ( $R G)$ se practicó un análisis de varianza combinado. Los índices de estabilidad calculados fueron la desviación estándar $\left(\mathrm{S}_{\mathrm{i}}\right)$ y el coeficiente de variación $\left(\mathrm{CV}_{\mathrm{i}}\right)$ de Francis y Kannenberg, los parámetros de estabilidad $\left(b_{i} y\right.$ $\mathrm{S}^{2} \mathrm{~d}_{\mathrm{i}}$ ) de Eberhart y Russell, la ecovalencia $\left(\mathrm{W}_{\mathrm{i}}\right)$ de Wricke, la varianza de estabilidad $\left(\sigma_{i}^{2}\right)$ de Shukla, los índices no parámetricos $\left(\mathrm{S}_{\mathrm{i}}^{(1)}\right.$ y $\left.\mathrm{S}_{\mathrm{i}}^{(2)}\right)$ de Huehn, y la medida de superioridad de un cultivar $\left(\mathrm{P}_{\mathrm{i}}\right)$ de Lin y Binns. Los resultados mostraron que Chalqueño, ETA 13, H-40, San Lucas y VS-46E tuvieron los índices de estabilidad más pequeños. La metodología del biplot identificó a estos 5 genotipos, así como a Ixtlahuaca y HIT-3 como variedades estables y de alto rendimiento (de 5,92 a 7,91 t.ha-1). La metodología del biplot indicó que $\mathrm{RG}, \mathrm{b}_{\mathrm{i}}, \mathrm{P}_{\mathrm{i}}$ y $\mathrm{CV}_{\mathrm{i}}$, estuvieron relacionados, pero tuvieron poca o ninguna relación con $\mathrm{S}_{\mathrm{i}}, \mathrm{W}_{\mathrm{i}}, \sigma_{\mathrm{i}}^{2}, \mathrm{~S}_{\mathrm{i}}^{(1)}, \mathrm{S}_{\mathrm{i}}^{(2)}$ y $\mathrm{S}^{2} \mathrm{~d}_{\mathrm{i}}$. Chalqueño y ETA

1 Autor para correspondencia. Correo electrónico: agonzalezh@uaemex.mx

Facultad de Ciencias Agrícolas, Centro de Investigación y Estudios Avanzados en Fitomejoramiento, Universidad Autónoma del
Aceptado: $24 / 08 / 10$

\section{ABSTRACT}

Aplication and comparison of univariate methods to evaluate the maize stability at Toluca-Atlacomulco Valley, Mexico. The objective of this study was to apply 6 univariate methods to evaluate the stability of 25 maize (Zea mays L.) genotypes of Mexico's highlands. The genotypes were evaluated in 4 environments, under a randomized complete block design, with 4 replications per environment. A combined analysis of variance with grain yield data was performed. The stability indexes calculated were Francis and Kannenberg's standard deviation $\left(\mathrm{S}_{\mathrm{i}}\right)$ and coefficient of variation $\left(\mathrm{CV}_{\mathrm{i}}\right)$, Eberhart and Russell's stability parameters $\left(b_{i}\right.$ and $\left.S^{2} d_{i}\right)$, Wricke's ecovalence $\left(\mathrm{W}_{\mathrm{i}}\right)$, Shukla's stability variance $\left(\sigma_{i}^{2}\right)$, Huehn's nonparametric measures $\left(\mathrm{S}_{\mathrm{i}}^{(1)}\right.$ and $\left.\mathrm{S}_{\mathrm{i}}^{(2)}\right)$, and Lin and Binns' assessment of cultivar superiority $\left(\mathrm{P}_{\mathrm{i}}\right)$. The results showed that almost all genotypes were classified as stable with 6 methods; Chalqueño, ETA 13, H-40, San Lucas, and VS-46E had lowest stability indexes. The biplot methodology identified these 5 genotypes, as well as Ixtlahuaca and HIT-3, as high yielding and stable varieties (from 5,92 to 7,91 t.ha-1). The biplot methodology also indicated that $R G, b_{i}, P_{i}$ y $C_{i}$ were correlated,

Estado de México, Campus Universitario El Cerrillo Piedras Blancas, Toluca, Estado de México. en Olericultura, Departamento de Fitotecnia, Universidad Autónoma Chapingo, Estado de México. 
13 pertenecen a la raza Chalqueño, Ixtlahuaca y San Lucas fueron identificadas en la raza Cónico, mientras que H-40, HIT-3 y VS-46E tienen germoplasma de otras razas, por lo que éstos podrían emplearse en un programa de mejoramiento para incrementar el rendimiento de grano y la estabilidad de los maíces de esta región de México.

\section{INTRODUCCIÓN}

El entendimiento de la relación entre el comportamiento del cultivo (el fenotipo observado) y el ambiente ha sido fundamental para los fitomejoradores y genetistas. El comportamiento de una variedad es una función del genotipo $(\mathrm{G})$, del ambiente (A) y de la interacción GxA (IGA). La IGA, entendida como el comportamiento relativo que muestran los genotipos a través de condiciones ambientales diversas, causa confusión en la estimación de parámetros genéticos, reduce la respuesta a la selección y dificulta la identificación de genotipos superiores; no obstante, su análisis e interpretación permite identificar mega-ambientes, detectar genotipos con adaptación amplia o específica, proponer estrategias para su mejoramiento genético o generar tecnología (Márquez 1992, Crossa 1990, Rodríguez et al. 2002).

En el Valle Toluca-Atlacomulco, México, se siembran 250000 ha de maíz y un $85 \%$ de esta superficie se establece comercialmente en condiciones de secano y el otro $15 \%$ en punta de riego y humedad residual. El $87 \%$ de los agricultores en esta región usan semilla criolla proveniente de las razas Cónico, Chalqueño, Palomero Toluqueño y Cacahuacintle, con rendimientos de grano entre 2,5 y 6,5 t.ha ${ }^{-1}$ (Niño et al. 1998, Herrera et al. 2002), pero actualmente se han liberado híbridos que tienen rendimientos de grano potencial hasta de 12,0 t.ha ${ }^{-1}$; sin embargo en la literatura especializada existe poca but they had little or no association with $\mathrm{S}_{\mathrm{i}}, \mathrm{W}_{\mathrm{i}}$, $\sigma_{i}^{2}, S_{i}^{(1)}, S_{i}^{(2)}$ and $S^{2} d_{i}$ indexes. Chalqueño and ETA 13 are Chalqueño race, Ixtlahuaca and San Lucas were identified as Cónico race, whereas and H-40, HIT-3, and VS-46E belong to other races, so these genotypes could be employed for increased the grain yield and the stability of maize in this region of Mexico.

información sobre antecedentes de su potencial productivo, de la raza a la que pertenecen, de sus relaciones de parentesco, de su estabilidad y de las relaciones entre índices de estabilidad, que permita recomendar confiablemente la siembra de genotipos sobresalientes aún en las áreas con gran variación ambiental (González et al. 2006). En el contexto anterior se planteó este estudio para identificar genotipos de alto rendimiento y estabilidad y determinar la relación entre los índices de estabilidad aplicados a maíces del Valle Toluca-Atlacomulco, Estado de México.

\section{MATERIALES Y MÉTODOS}

\section{Descripción del área experimental}

Este estudio se realizó en el año 2004 en 4 ambientes de los Valles Altos del Centro de México, representados por El Cerrillo Piedras Blancas (2 fechas de siembra), Metepec y Jocotitlán, localidades situadas en el Valle TolucaAtlacomulco, estado de México. El Cerrillo está localizado a $19^{\circ} 17^{\prime}$ de latitud norte (LN), 99³9' de longitud oeste (LO), a una altitud de $2640 \mathrm{~m}$, su clima es templado húmedo, con suelo vertisol y precipitación media anual (PMA) de $790 \mathrm{~mm}$. Metepec está situada a $19^{\circ} 13^{\prime}$ de LN, $99^{\circ} 35^{\prime}$ de LO, a una altitud de $2606 \mathrm{~m}$, su clima es templado húmedo, con PMA de $980 \mathrm{~mm}$ y tiene suelo andosol. Jocotitlán se sitúa a $19^{\circ} 42^{\prime}$ de LN, 9943' de LO, a una altura de $2553 \mathrm{~m}$, su clima 
es templado seco, presenta suelo planosol mólico y su PMA es de 669 mm (González et al. 2008).

\section{Material genético}

Se consideraron 25 genotipos: 12 criollos que podrían ser representativos de las 4 razas descritas por Wellhausen et al. (1951) y 13 híbridos formados con germoplasma de esas y otras razas de Valles Altos del Centro de México (Cuadro 1;
González et al. 2008). En la literatura especializada han sido documentadas Cacahuacintle 5 (González et al. 2006), H-33 (Gámez et al. 1996), H-40 (Velásquez et al. 2005), e Ixtlahuaca (Niño et al. 1998, Nava y Mejía 2002).

\section{Diseño y tamaño de la parcela experimental}

Los 25 genotipos de maíz fueron evaluados en 4 experimentos conducidos en campo bajo

Cuadro 1. Material genético según raza utilizado en el presente estudio, por zona geográfica.

\begin{tabular}{|c|c|c|c|c|}
\hline $\mathrm{N}^{\circ}$. & Población & Municipio de colecta & \multicolumn{2}{|l|}{ Raza } \\
\hline 4-9 & Cacahuacintle 1 a 6 & Valle de Toluca & \multicolumn{2}{|c|}{ Cacahuacintle } \\
\hline 20 & Ixtlahuaca & Ixtlahuaca & \multicolumn{2}{|l|}{ Cónico } \\
\hline 23 & San Lucas & Metepec & \multicolumn{2}{|l|}{ Cónico } \\
\hline 11 & Chalqueño & Chalco-Amecameca & \multicolumn{2}{|l|}{ Chalqueño } \\
\hline 12 & ETA 13 & Jocotitlán & \multicolumn{2}{|l|}{ Chalqueño } \\
\hline 21 & Negro & Jiquipilco & \multicolumn{2}{|l|}{ Chalqueño } \\
\hline 22 & Palomero Toluqueño & Valle de Toluca & \multicolumn{2}{|c|}{ Palomero Toluqueño } \\
\hline & & \multicolumn{3}{|l|}{ Origen de los híbridos } \\
\hline $\mathrm{N}^{\mathrm{o}}$. & Híbrido & Genealogía ${ }^{J}$ & Institución & Híbrido \\
\hline 14 & $\mathrm{H}-33$ & (M28xM27)x(M18xM17) & INIFAP & Doble \\
\hline 15 & $\mathrm{H}-40$ & (CML246xCML242)xM39 & INIFAP & Triple \\
\hline 16 & $\mathrm{H}-44$ & (M46xM47)xM48 & INIFAP & Triple \\
\hline 17 & H-90E (H-52) & (M43xM44)xM48 & INIFAP & Triple \\
\hline 18 & HIT-3 & (CML239xCML242)xCML349 & ICAMEX & Triple \\
\hline 19 & HIT-7 & (CML239xCML242)xIML-6 & ICAMEX & Triple \\
\hline 10 & Cóndor & No disponible & ASGROW & Triple \\
\hline 13 & Gavilán & No disponible & ASGROW & Doble \\
\hline 1 & AS-721 & No disponible & ASPROS & Doble \\
\hline 2 & AS-722 & No disponible & ASPROS & Triple \\
\hline 3 & AS- 820 & No disponible & ASPROS & Triple \\
\hline 25 & Z-60 & No disponible & ASGROW & Triple \\
\hline 24 & VS-46E & No disponible & INIFAP & Sintético \\
\hline
\end{tabular}

Fuente: González et al. (2008). 
un diseño experimental de bloques completos al azar; se emplearon 4 repeticiones para cada uno de los 4 experimentos. La parcela experimental era de 3 surcos de 6,0 m de longitud y $0,80 \mathrm{~m}$ de ancho; en cada surco hubo 33 plantas distanciadas a $0,20 \mathrm{~m}$ (62 500 plantas.ha $\left.^{-1}\right)$; el surco central fue la unidad experimental útil (UEU). En el contexto anterior, los bloques son sinónimo de repeticiones. El propósito del bloqueo es reducir la heterogeneidad ambiental dentro del experimento, al agrupar a las unidades experimentales de una misma repetición en una franja de terreno uniforme; para que el diseño experimental sea eficiente debe haber diferencias significativas entre bloques.

\section{Manejo agronómico}

Los 4 experimentos se sembraron manualmente los días 7 (Jocotitlán, con riego de presiembra), 12 (El Cerrillo, fecha 1 con riego de presiembra), 14 (Metepec) y 26 (El Cerrillo, fecha 2) de abril de 2004. La fertilización se hizo con el tratamiento $140 \mathrm{~N}-80 \mathrm{P}-30 \mathrm{~K}$; en la siembra se aplicó el $50 \%$ del nitrógeno y todo el fósforo y el potasio y el otro $50 \%$ del nitrógeno se incorporó con la segunda escarda. Se manejaron 62500 plantas.ha ${ }^{-1}$. La maleza se controló mecánica y químicamente; en este último caso se aplicó 1,0 1 y $1,0 \mathrm{~kg} \cdot \mathrm{ha}^{-1}$ de $2,4-\mathrm{D}$ amina y atrazina, respectivamente disueltos en 2001 de agua.

\section{Análisis estadístico}

El rendimiento de grano (RG) en cada uno de los 4 experimentos se calculó con las plantas cosechadas en la UEU. Posteriormente se ajustó por plantas faltantes con la formula de Iowa (González et al. 2008) y por humedad (14\%) y se registró en t.ha ${ }^{-1}$. Las técnicas estadísticas empleadas fueron el análisis de varianza individual y el que resultó de combinar la información de los 4 experimentos. Las hipótesis relacionadas con los análisis de varianza se probaron con los procedimientos descritos por Sahagún (1998). La comparación de medias entre genotipos para cada ambiente y para los promedios aritméticos a través de éstos se hizo con la prueba de Tukey al nivel de significancia del 1\%; los procedimientos para su cálculo fueron descritos por Martínez (1988). Estos análisis estadísticos se obtuvieron con el paquete Sistema para Análisis Estadístico (Statistical Analysis System, SAS) versión 6.11 para Windows.

Los datos fueron sometidos a las pruebas de normalidad de Shapiro y Wilk y la de homogeneidad de varianzas de Bartlett (Steel y Torrie 1980), antes de aplicar los métodos univariados para estudiar la interacción genotipo $\mathrm{x}$ ambiente y la estabilidad de los genotipos de maíz. Los métodos utilizados fueron la desviación estándar $\left(\mathrm{S}_{\mathrm{i}}\right)$ y el coeficiente de variación $\left(\mathrm{CV}_{\mathrm{i}}\right)$ de Francis y Kannenberg (1978), los parámetros de estabilidad $\left(b_{i}\right.$ y $\left.S^{2} d_{i}\right)$ de Eberhart y Russell (1966), la ecovalencia $\left(\mathrm{W}_{\mathrm{i}}\right)$ de Wricke (1962), la varianza de estabilidad $\left(\sigma_{\mathrm{i}}^{2}\right)$ de Shukla (1972), los índices no paramétricos $\left(\mathrm{S}_{\mathrm{i}}^{(1)}\right.$ y $\left.\mathrm{S}_{\mathrm{i}}^{(2)}\right) \mathrm{de}$ Huehn (Nassar y Hühn 1987) y la medida de superioridad de un cultivar $\left(\mathrm{P}_{\mathrm{i}}\right)$ de Lin y Binns (1988); la aplicación se hizo en la forma como lo sugirieron Castañón y Tosquy (1996) y Rodríguez et al. (2002).

El análisis de varianza para un diseño experimental de bloques completos al azar para evaluar genotipos en varios ambientes se fundamenta en el siguiente modelo (Sahagún 1998):

$$
\mathrm{Y}_{\mathrm{ijk}}=\mu+\mathrm{A}_{\mathrm{j}}+\mathrm{R}(\mathrm{A})_{\mathrm{k}(\mathrm{j})}+\mathrm{G}_{\mathrm{i}}+(\mathrm{GA})_{\mathrm{ij}}+\varepsilon_{\mathrm{ijk}}
$$

Donde: $\mu$ es la media general; $G_{i}, A_{j} y$ $(\mathrm{GA})_{\mathrm{ij}}$ son los efectos de genotipos, ambientes e interacción genotipo $\mathrm{x}$ ambiente, respectivamente; $\mathrm{R}(\mathrm{A})_{\mathrm{k}(\mathrm{j})}$ es el efecto de repeticiones anidadas dentro de ambientes; y $\varepsilon_{\mathrm{ijk}}$ el error experimental de la ijk-ésima observación.

$\mathrm{Si}$ genotipos es considerado como factor de efectos fijos y ambientes y repeticiones como factores de efectos aleatorios, las pruebas de F son iguales a las presentadas por Sahagún (1998), para el modelo con restricciones sobre los parámetros de efectos fijos (genotipos).

La desviación estándar y el coeficiente de variación de los genotipos representan el nivel de 
adaptación de éstos a condiciones ambientales variables, sin que se incluya el efecto ambiental; valores pequeños de ambos estadísticos representan mayor estabilidad (Francis y Kannenberg 1978).

El índice de superioridad de Lin y Binns (1988) surgió de la necesidad de contar con una medida de estabilidad cuando el experimento o las variedades testigo no se pueden manejar en todos los ambientes. Este índice se calcula a partir de la suma de cuadrados de las diferencias entre el genotipo de interés con respecto al genotipo de mayor rendimiento en cada uno de los ambientes, por lo que representa el cuadrado medio del efecto conjunto de genotipos e interacción GxA y, al calcularse con referencia a la máxima respuesta, determina la adaptabilidad en un sentido general.

La ecovalencia $\left(\mathrm{W}_{\mathrm{i}}\right)$ de Wricke (1962) es uno de los índices más sencillos para estimar la estabilidad fenotípica; a partir de éste se determina la contribución de cada genotipo a la interacción GxA. Los genotipos con valores de $\mathrm{W}_{\mathrm{i}}$ pequeños son considerados como estables. Este índice y la varianza de estabilidad de Shukla (1972) clasifican a los genotipos de manera similar.

La varianza de estabilidad de Shukla (1972) se basa en la descomposición de la interacción GxA en g genotipos; ésta es igual a la varianza ambiental dentro más la varianza ambiental entre para cada genotipo, corregida por efectos aditivos de ambientes. El genotipo será clasificado como estable cuando ambas variables sean igual a cero. Una extensión del método involucra una varianza que incluye las desviaciones de regresión y el error residual; además se consideró como covariable el efecto ambiental. Esta metodología determina la estabilidad en el sentido estático (Lin et al. 1986).

Los índices no paramétricos de Huehn (Nassar y Hühn 1987) se basan en la asignación de rangos por ambientes, después se calcula la suma de diferencias absolutas de los rangos del genotipo i con respecto a otros genotipos a través de los ambientes y la varianza entre rangos del genotipo i sobre ambientes. En ambas metodologías, una variedad es clasificada como estable si sus rangos son similares a través de ambientes y presentan mínima varianza. Al no depender de distribuciones probabilísticas, ambos índices son fáciles de calcular e interpretar y se pueden aplicar a experimentos desbalanceados. Se pueden realizar pruebas estadísticas aproximadas para ambos índices, basadas en la distribución normal. En un inicio se realizó una prueba global que consideró los índices de los i genotipos mediante una prueba de Chi-cuadrada; si ésta es significativa se realiza el análisis individual de los genotipos.

Los parámetros de estabilidad de Eberhart y Russell (1966) incluyen al coeficiente de regresión y a la varianza de las desviaciones de regresión de cada genotipo; el primero se calcula con base en el efecto ambiental (índice ambiental), considerado fijo, y representa la respuesta de un genotipo al mejorar la condición ambiental, mientras que el segundo representa una medida de variabilidad, interpretada como falta de estabilidad. Un genotipo es estable si su media es superior al promedio general, su coeficiente de regresión es igual a 1 y sus desviaciones de regresión son iguales a 0 .

En el Cuadro 2 se muestran las formulas para estimar cada uno de los índices de estabilidad. La significancia estadística de los índices de Wricke (1962), de Shukla (1972) y de Eberhart y Russell (1966) se determinaron con una prueba de F, mientras que los de Huehn (Nassar y Hühn 1987) se hizo con base en una prueba de Chicuadrada. La significancia de los coeficientes de regresión $\left(b_{\mathrm{i}}\right)$ también se puede determinar con una prueba de $t$.

El rendimiento de grano $(\mathrm{RG})$ y los índices de estabilidad univariados fueron sometidos a un análisis de componentes principales (ACP), donde se aplico la técnica descrita por Sánchez (1995). El RG fue incluido junto con los índices de estabilidad en la gráfica del biplot debido a que en México los agricultores han incrementado la producción en sus variedades criollas al 
Cuadro 2. Índice de estabilidad fenotípica considerados.

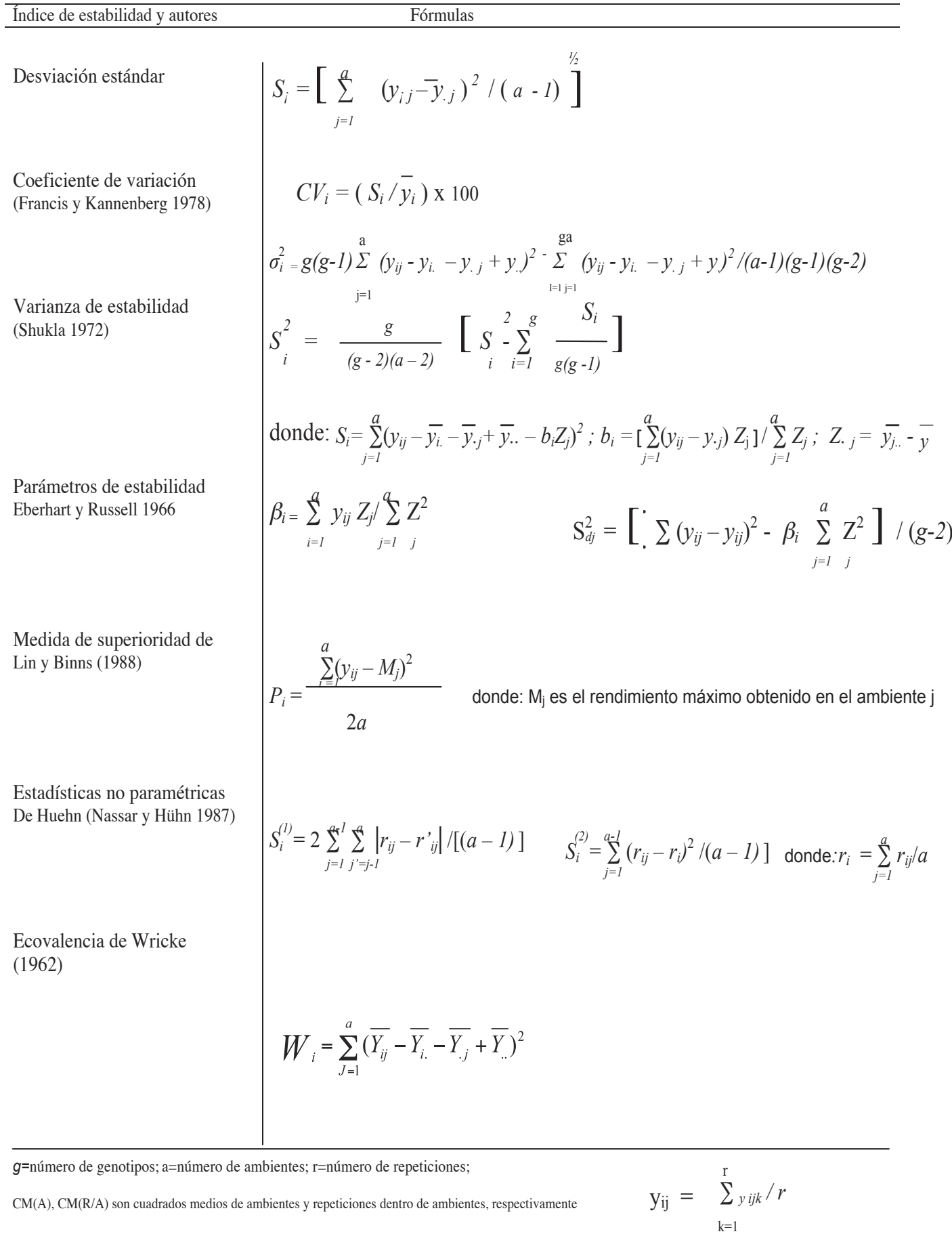


seleccionar indirectamente los componentes del rendimiento asociado a las características de la mazorca, como longitud, diámetro, proporción maíz/olote, número de hileras con grano, color del grano, tipo de grano y sanidad de mazorca, principalmente; además, se propuso estudiar la interrelación entre RG y los índices de estabilidad, para identificar genotipos de maíz de alto rendimiento y mínima interacción genotipo $\mathrm{x}$ ambiente. Antes de obtener el biplot, los datos fueron estandarizados y su estructura de correlaciones fue sometida a la descomposición de valores singulares. La estandarización se hizo al restar la columna de medias (variables) y posteriormente al dividir los elementos de cada columna por la raíz cuadrada de la suma de cuadrados de desviaciones. En el biplot se analiza la estructura de una matriz de datos (varianzas y correlaciones) compuesta de unidades taxonómicas (genotipos), asignadas a las hileras, y los valores de las variables que los caracterizan (rendimiento e índices de estabilidad), asignados a las columnas. El biplot reemplazó la necesidad de usar gráficas múltiples, análisis de agrupamiento y cuadros de coeficientes de correlación, al permitir la determinación visual y confiable de la existencia de patrones entre las unidades taxonómicas como resultado de los valores de sus variables, que variables separan los grupos definidos y que relación existe entre las variables. La similitud entre unidades taxonómicas o entre variables se puede determinar por la magnitud del ángulo que se forma entre parejas de vectores; un ángulo de $90^{\circ}$ indica no correlación; un ángulo de $0^{\circ}$ ó de $180^{\circ}$ indica correlación de 1,0 ó -1,0, respectivamente. En un mismo cuadrante del biplot, puntos cercanos tienen valores similares y viceversa. Los resultados del ACP se obtuvieron con el programa escrito en lenguaje matricial interactivo del SAS para sistema operativo DOS, descrito por Sánchez (1995). La gráfica del biplot se hizo en Microsoft Excel, y se consideraron las coordenadas de los genotipos y de las variables correspondientes a los 2 primeros componentes principales.

\section{RESULTADOS Y DISCUSIÓN}

\section{Análisis de varianza (anava) y comparación de medias}

$\mathrm{El}$ anava para rendimiento de grano detectó diferencias significativas entre ambientes (A), entre genotipos $(\mathrm{G})$ y en la interacción GxA (Cuadro 3); la contribución de cada una de estas fuentes a la variabilidad total del modelo fue de 45,1 , 48,4 y $6,5 \%$, respectivamente. Estos resultados indican que al menos entre 2 ambientes o entre 2 genotipos existieron diferencias reales en la (s) característica (s) a evaluar y, adicionalmente, que el comportamiento de los genotipos varío en función del ambiente. La significancia de la IGA sugiere que algunos genotipos de maíz tuvieron

Cuadro 3. Análisis de varianza combinado para rendimiento de grano evaluado en 4 ambientes del Valle Toluca-Atlacomulco. Estado de México, México, 2004.

\begin{tabular}{lcccc}
\hline Fuente de variación & Grados de libertad & Cuadrados medios & Valor de F & \% del modelo \\
\hline Ambientes (A) & 3 & 195,44 & $291,7 * *$ & 45,1 \\
Repeticiones / A & 12 & 1,03 & & 48,4 \\
Genotipos (G) & 24 & 26,21 & $22,40^{* *}$ & 6,5 \\
Interacción GxA & 72 & 1,17 & $1,74 * *$ & \\
Error combinado & 288 & 0,67 & & \\
\hline
\end{tabular}

Media aritmética general=6,37 t.ha ${ }^{-1}$; coeficiente de variación=12,8\%; **=significativo con $\mathrm{p}=0,019^{9}$ Modelo=suma de cuadrados de ambientes, variedades e interacción GxA; $\mathrm{R}^{2}$ del modelo general=87,0\%. 
mejor adaptación a un ambiente específico, lo que podría limitar la identificación de genotipos superiores para diferentes condiciones ambientales, situación muy común para los Valles Altos del Centro de México, donde existe gran heterogeneidad ambiental. En este punto el empleo de técnicas que permitan al fitomejorador seleccionar materiales de mayor rendimiento y estabilidad cobra relevancia (Rodríguez et al. 2002), ya que éste es uno de los objetivos más importantes en los programas de mejoramiento genético.

González et al. (2008) mencionaron que en los Valles Altos del Centro de México existe heterogeneidad ambiental relacionada con altitud, temperatura, precipitación y tipo de suelo. En Jocotitlán predomina el clima templado seco, mientras que en El Cerrillo y Metepec el templado húmedo; las diferencias en altitud entre Jocotitlán y Metepec son de $53 \mathrm{~m}$, y entre la primera y El Cerrillo son de $87 \mathrm{~m}$. Las precipitaciones medias anuales para Jocotitlán, El Cerrillo y Metepec son de 669, 790 y $980 \mathrm{~mm}$, respectivamente, mientras que el tipo de suelo predominante, en el mismo orden, es planosol mólico, vertisol y andosol. En el 2004 en las estaciones climatológicas de El Cerrillo, Metepec y Jocotitlán, se registró una precipitación pluvial acumulada de 1114, 938 y 806 mm, respectivamente. Como estos valores fueron superiores al promedio anual de cada localidad y son adecuados para el desarrollo del maíz, es probable que las diferencias en rendimiento de grano entre ambientes se deban a que en El Cerrillo se presentaron problemas de drenaje causados por el tipo de suelo (vertisol), particularmente durante las épocas de floración y llenado de grano, lo que contribuyó a una disminución en el rendimiento de los 25 genotipos en relación a las otras localidades (Cuadro 3), debido principalmente a un incremento en los porcentajes de acame y pudrición de mazorca causada por Fusarium moniliforme.

Las diferencias en rendimiento entre genotipos (Cuadro 3) son atribuibles al tipo de variedad (criollo o híbrido) y probablemente, a la raza a la que pertenecen (particularmente en el caso de los criollos); los 12 criollos pertenecen a las razas
Palomero Toluqueño, Cacahuacintle, Cónico y Chalqueño, y los 13 híbridos tienen germoplasma de Cónico, Chalqueño y razas de otros países (González et al. 2008); el cambio de posición de los genotipos a través de los ambientes refleja la significancia de la IGA. Crossa (1990) y Sabaghnia et al. (2006) comentaron que la interacción cruzada dificulta la identificación de genotipos superiores. Los híbridos superaron a las variedades en 1,32 t.ha ${ }^{-1} \mathrm{y}$ los primeros respondieron mejor en las localidades de Metepec y Jocotitlán.

En la primera fecha de siembra plantada en El Cerrillo (A1), los híbridos superaron a los criollos en 0,91 t.ha- ${ }^{-1}$ (Cuadro 4). H-90E produjo el mayor rendimiento de grano $\left(7,71\right.$ t.ha $\left.^{-1}\right)$ y fue estadísticamente diferente de Z-60, HIT-7, AS.820, HIT-3, VS-46E, los 6 Cacahuacintles y Palomero Toluqueño (medias entre 3,77 y 5,21 t.ha $\left.^{-1}\right)$.

En la segunda fecha de siembra plantada en El Cerrillo (A2), los híbridos y los criollos tuvieron el mismo rendimiento de grano promedio (Cuadro 4). H-90E fue el mejor genotipo (7,09 t.ha $\left.\mathrm{a}^{-1}\right)$ y superó significativamente a Gavilán, AS-721, Z-60, HIT-7, AS-820, los 6 Cacahuacintles y a Palomero Toluqueño (medias entre 3,66 y 4,70 t.ha-1).

En Metepec (A3) la diferencia en el rendimiento de grano promedio entre los híbridos y los criollos fue de 2,06 t.ha-1 (Cuadro 4). H-90E tuvo el mayor rendimiento $\left(10,82\right.$ t.ha $\left.^{-1}\right)$ pero sólo difirió estadísticamente de AS-721, Z-60, HIT-3, San Lucas, Ixtlahuaca, VS-46E, los 6 Cacahuacintles y Palomero Toluqueño (promedios entre 4,02 y 7,78 t.ha-1).

En Jocotitlán (A4) los híbridos superaron, en promedio, a los criollos en 1,61 t.ha ${ }^{-1}$ (Cuadro 4). $\mathrm{H}-44$ fue el mejor genotipo $\left(9,58 \mathrm{t}^{-h^{-1}}\right)$ pero sólo se diferenció estadísticamente de AS-820, los 6 Cacahuacintles y Palomero Toluqueño (promedios entre 4,98 y 7,03 t.ha-1).

Los resultados anteriores indican que los genotipos con mayor rendimiento de grano a través de los 4 ambientes $(\mathrm{X})$ fueron $\mathrm{H}-90 \mathrm{E}, \mathrm{H}-44$, H-40, H-33, ETA 13, Cóndor, AS-722, Chalqueño y Negro (de 8,71 a 7,12 t.ha-1 ${ }^{-1}$ Cuadro 4). La 


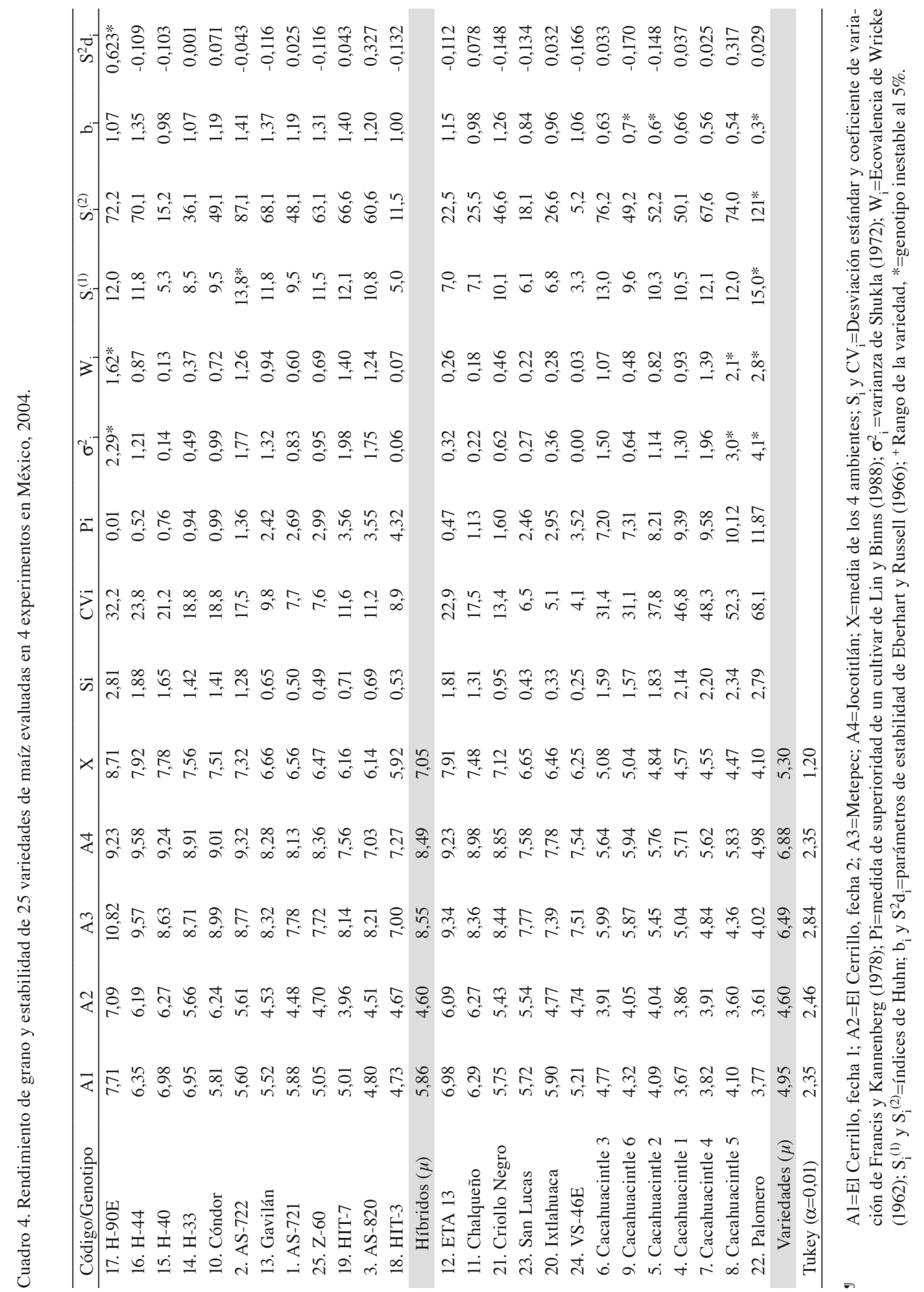


superioridad de H-90E se atribuye a que éste respondió muy bien en malos ambientes, como El Cerrillo en ambas fechas, y también lo hizo en buenos ambientes, como Metepec y Jocotitlán; en 3 ambientes se clasificó en primer lugar y en el otro (Jocotitlán) fue cuarto. ETA 13, Chalqueño, y Negro, de la raza Chalqueño, fueron los criollos más sobresalientes al producir 7,12; 7,48 y 7,91 t.ha ${ }^{-1}$, pero comparada con la raza Cónico, ETA 13 sólo difirió estadísticamente de San Lucas e Ixltahuaca (Cuadro 4). Niño et al. (1998) concluyeron que las variedades criollas identificadas como Ixtlahuaca y ETA 13 produjeron 6,42 y 6,45 t.ha ${ }^{-1}$. La superioridad de H-40 sobre H-33 fue detectada por Velásquez et al. (2005); en punta de riego, en humedad residual, y en temporal favorable, su diferencia fue de 1,58; 1,27 y 1,78 t.ha ${ }^{-1}$, respectivamente. Los rendimientos de los 6 Cacahuacintles se ubicaron dentro del intervalo proporcionado por González et al. (2006), que fue de 4,06 a 6,37 thä ${ }^{-1}$. La identificación de genotipos sobresalientes de maíz de grano azul ya había sido reportada por Arellano et al. (2003), quienes al evaluar variedades criollas de la raza Chalqueño en el Seco, Puebla, México, identificaron criollos con rendimientos de grano entre 8,7 y 10,5 t.ha ${ }^{-1}$, y al promediar entre ambientes, el mejor de ellos produjo 6,4 t.ha ${ }^{-1}$.

Aún cuando Chalqueño y Cónico superaron estadísticamente a Palomero Toluqueño $(4,10$ t.ha ${ }^{-1}$ ) y Cacahuacintle (entre 4,47 y 5,08 t.ha-1), las 2 últimas, por presentar granos reventadores o harinosos, respectivamente, tienen mayor demanda y un precio hasta 6 veces mayor (Arellano et al. 2003, González et al. 2006). La importancia de Palomero Toluqueño y Cacahuacintle también se ha reflejado en los programas de mejoramiento genético de Valles Altos de México, ya que fueron los progenitores de Cónico y Chalqueño, las razas predominante y de mayor rendimiento, respectivamente (Wellhausen et al. 1951).

\section{Índices de estabilidad}

Los 6 métodos clasificaron a la mayoría de las variedades como estables o deseables (Cuadro 4).
Al aplicar los métodos de Shukla (1972) y Wricke (1962) se observó (Cuadro 4) que los genotipos identificados como inestables al 5\% fueron H-90E, Cacahuacintle 5 y Palomero Toluqueño; el resto se clasificaron como estables, aunque de ellos, VS-46E, HIT-3, H-40, Chalqueño, San Lucas y ETA 13 fueron los que presentaron las varianzas de estabilidad $\left(\sigma_{\mathrm{i}}^{2}\right)$ y las ecovalencias $\left(\mathrm{W}_{\mathrm{i}}\right)$ más pequeñas.

Con los índices de Huehn $\left(\mathrm{S}_{i}^{(1)}\right.$ y $\left.\mathrm{S}_{i}^{(2)}\right)$ se detectó que sólo Palomero Toluqueño y AS-722 fueron inestables $(\mathrm{p}<0,05)$. VS-46E, HIT-3, H-40, San Lucas, Ixtlahuaca, Chalqueño y ETA 13 presentaron los índices más pequeños (Cuadro 4).

En el método de Eberhart y Russell (1966) se observó que los Cacahuacintles 6 y $2\left(\mathrm{~b}_{\mathrm{i}}\right)$ y H-90E $\left(\mathrm{S}^{2} \mathrm{~d}_{\mathrm{i}}\right)$ fueron inestables $(\mathrm{p}<0,05)$. Los genotipos deseables, con coeficientes y desviaciones de regresión cercanos a 1 y 0 , respectivamente, fueron Chalqueño, ETA 13, H-33, H-40, HIT-3, Ixtlahuaca y VS-46E (Cuadro 4).

Los 2 índices de Francis y Kannenberg (1978) produjeron resultados similares (Cuadro 4); los genotipos estables o deseables, con rendimiento alto $(\mathrm{RG})$ y coeficiente de variación $(\mathrm{CV})$ bajo fueron Ixtlahuaca, Z-60, San Lucas, AS-721, Gavilán, Negro, AS-722, Chalqueño, Cóndor, H-33, y H-40.

Con la medida de superioridad de un cultivar $\left(\mathrm{P}_{\mathrm{i}}\right)$ de Lin y Binns (1988) se detectó que Z-60, Ixtlahuaca, AS-721, San Lucas, Gavilán, Negro, AS-722, Cóndor, Chalqueño, H-33, H-40, ETA 13, H-44 y H-90E tuvieron los mayores rendimientos e índices pequeños de $\mathrm{P}_{\mathrm{i}}$ (Cuadro 4).

De los resultados anteriores se puede concluir que los 6 métodos de estabilidad univariados identificaron a Chalqueño, ETA 13, H-40, San Lucas y VS-46E como los genotipos con los valores más pequeños y, por lo tanto, los más deseables. A excepción de VS-46E, las otras 4 variedades tuvieron rendimientos mayores que el promedio general $\left(6,37\right.$ t.ha $\left.^{-1}\right)$.

\section{Análisis de componentes principales}

La estabilidad estática o biológica está relacionada con varianzas pequeñas para rendimiento 
entre ambientes, pero no es deseable ya que un genotipo así no responde a la aplicación de mayores insumos o a mejoras agronómicas o del ambiente. Por otra parte, la estabilidad dinámica o agronómica implica que un genotipo es estable porque su comportamiento en cada ambiente corresponde al nivel estimado, por lo que este concepto es de gran importancia para los agrónomos y fitomejoradores, quienes prefieren genotipos de alto rendimiento y con el potencial de responder a la aplicación de más insumos o a mejoras ambientales (Sabaghnia et al. 2006).
El análisis de componentes principales aplicado al conjunto de datos integrado por rendimiento de grano y los 6 índices de estabilidad indicó que los componentes $1(60,0 \%)$ y 2 $(21,7 \%)$ explicaron casi un $82,0 \%$ de la variación original (Cuadro 5). Estos resultados sugieren que las correlaciones aproximadas que se pueden detectar en el biplot pueden interpretarse confiablemente (Sánchez 1995). Al analizar la distribución de los 25 genotipos de maíz (Cuadro 6) en el biplot de la Figura 1 se identificaron 2 grupos: el primero incluyó a Palomero Toluqueño

Cuadro 5. Valores característicos del análisis de componentes principales y proporción que explican de la variación total original.

\begin{tabular}{lccccccccccc}
\hline Componente principal & 1 & 2 & 3 & 4 & 5 & 6 & 7 & 8 & 9 & 10 \\
\hline Valores característicos & 5,998 & 2,171 & 0,987 & 0,602 & 0,139 & 0,085 & 0,01 & 0,003 & 0 & 0 \\
Contribución (\%) & 59,98 & 21,71 & 9,87 & 6,02 & 1,39 & 0,85 & 0,1 & 0,03 & 0 & 0 \\
\hline
\end{tabular}

Cuadro 6. Coordenadas para los genotipos de maíz en el biplot.

\begin{tabular}{lrc}
\hline Código/Genotipo de maíz & CP1 & CP2 \\
\hline 1. AS-721 & $-0,2648$ & 0,1005 \\
2. AS-722 & 0,0778 & 0,4547 \\
3. AS-820 & 0,0904 & 0,3395 \\
4. Cacahuacintle 1 & 0,4250 & $-0,3874$ \\
5. Cacahuacintle 2 & 0,2880 & $-0,3989$ \\
6. Cacahuacintle 3 & 0,4119 & $-0,1644$ \\
7. Cacahuacintle 4 & 0,6453 & $-0,2997$ \\
8. Cacahuacintle 5 & 0,9458 & $-0,0769$ \\
9. Cacahuacintle 6 & 0,0947 & $-0,3973$ \\
10. Cóndor & $-0,1756$ & 0,2301 \\
11. Chalqueño & $-0,4154$ & $-0,0684$ \\
12. ETA 13 & $-0,4090$ & 0,0120 \\
13. Gavilán & $-0,1316$ & 0,2314 \\
14. H-33 & $-0,3052$ & 0,0786 \\
15. H-40 & $-0,4714$ & $-0,1152$ \\
16. H-44 & $-0,0652$ & 0,3413 \\
17. H-90E & 0,3726 & 0,7388 \\
18. HIT-3 & $-0,5130$ & $-0,3438$ \\
19. HIT-7 & 0,0551 & 0,3051 \\
20. Ixtlahuaca & $-0,4395$ & $-0,1434$ \\
21. Criollo Negro & $-0,3146$ & 0,0960 \\
22. Palomero Toluqueño & 1,4245 & $-0,1212$ \\
23. San Lucas & $-0,4912$ & $-0,2425$ \\
24. VS-46E & $-0,6793$ & $-0,3374$ \\
25. Z-60 & $-0,1552$ & 0,1684 \\
\hline
\end{tabular}




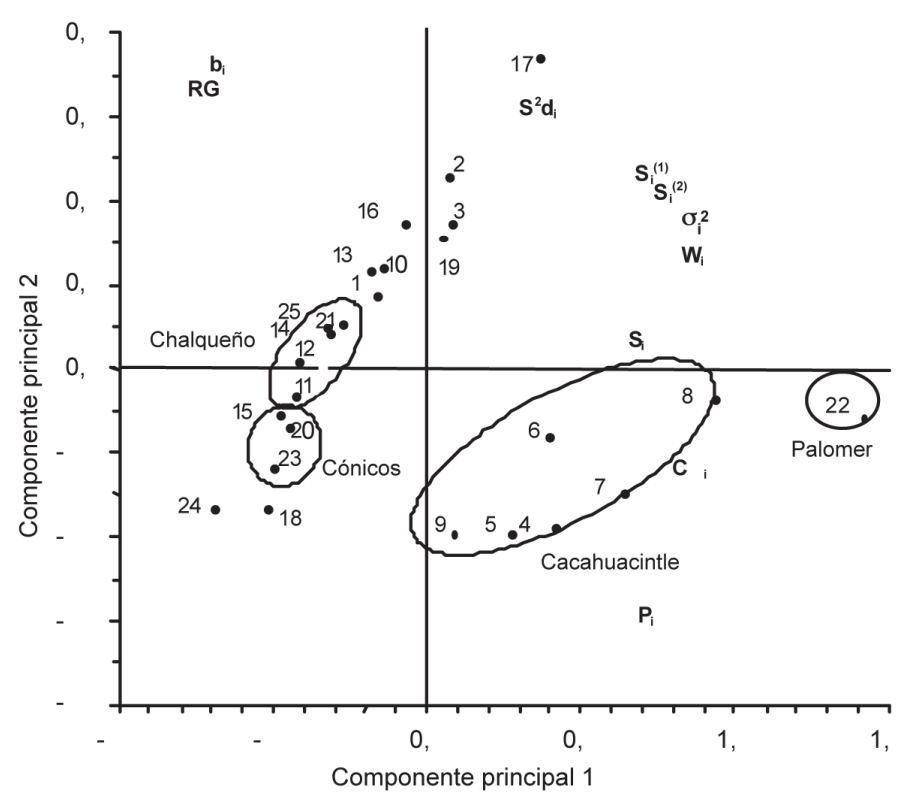

Fig. 1. Biplot entre el rendimiento de grano (RG) de 25 genotipos (identificados con número) y 6 índices de estabilidad (letras con superíndices y subíndices) aplicados a maíces de Valles Altos del Centro de México.

(22), Cacahuacintle (4 a 9), Cónico (20 y 23), Chalqueño (11), H-40 (15) y HIT-3 (18), mientras que en el otro grupo se localizó a ETA 13 (12) y Negro (21), de la raza Chalqueño, y a la mayoría de los híbridos formados con germoplasma de las razas Cónico, Chalqueño y otras. La raza Chalqueño (Cónico x Tuxpeño), empleada para derivar líneas que formaron al mejor genotipo (H-90E), así como las combinaciones entre líneas de esta raza y del CIMMYT, derivadas de germoplasma templado de Valles Altos de otros países, como H-44 (7,92 t.ha-1), podrían contribuir a una mejor explotación de la heterosis interracial en los Valles Altos del Centro de México.

La CP1 separó a RG y $b_{i}$ del resto de los índices (Figura 1, Cuadro 7). Flores et al. (1998) y Sabaghnia et al. (2006) cuando emplearon otros métodos de componentes principales observaron resultados similares. En el biplot también se observó que $\mathrm{S}_{\mathrm{i}}, \mathrm{S}_{\mathrm{i}}^{(1)}, \mathrm{S}_{\mathrm{i}}^{(2)}, \sigma_{\mathrm{i}}^{2}, \mathrm{~W}_{\mathrm{i}}$ y $\mathrm{S}^{2} \mathrm{~d}_{\mathrm{i}}$ estuvieron estrechamente relacionados, por lo que presentaron gran similitud en la definición de genotipos.
Sabaghnia et al. (2006) concluyeron que los índices que no tuvieron relación con RG, como $\mathrm{S}_{\mathrm{i}}, \mathrm{S}_{\mathrm{i}}^{(1)}$, $S_{i}^{(2)}, \sigma_{i}^{2}, W_{i}$ y $S^{2} d_{i}$, permiten identificar genotipos de alta estabilidad y rendimientos aceptables, pero los índices que están correlacionados negativamente con $\mathrm{RG}$, como $\mathrm{CV}_{\mathrm{i}}$ y $\mathrm{P}_{\mathrm{i}}$, no deben ser recomendados para la selección de cultivares superiores; esto último parece una contradicción ya que la recomendación es elegir genotipos con el mayor rendimiento e índices de estabilidad pequeños.

En la Figura 1 también se observó que $\mathrm{S}_{\mathrm{i}}$ y $\mathrm{CV}_{\mathrm{i}}$ clasificaron a los genotipos de manera similar, lo cual se explica por el hecho de que el primero es el numerador en la fórmula del $\mathrm{CV}_{\mathrm{i}}$. Kang et al. (1987) mostraron que $\sigma^{2}{ }_{i}$ y $W_{i}$, son, matemáticamente, el mismo índice y recomendaron al primero debido a la simplicidad en su cálculo. Los índices $\mathrm{S}_{\mathrm{i}}^{(1)}$ y $\mathrm{S}_{\mathrm{i}}^{(2)}$ se basan en rangos y están estrechamente asociados, pero el primero es más fácil de usar y permite una interpretación clara y relevante (Huehn 1990, Flores et al. 1998, Sabaghnia et al. 2006). En trigo (Triticum 
Cuadro 7. Coordenadas para las variables representadas en el biplot.

\begin{tabular}{lcc}
\hline Variables representadas/código & $\mathrm{CP1}$ & $\mathrm{CP} 2$ \\
\hline Rendimiento de grano $(\mathrm{RG})$ & $-0,6372$ & 0,6484 \\
Desviación estándar $\left(\mathrm{S}_{\mathrm{i}}\right)$ & 0,7423 & 0,0545 \\
Coeficiente de variación $\left(\mathrm{CV}_{\mathrm{i}}\right)$ & 0,8955 & $-0,2335$ \\
Medida de superioridad de un cultivar $\left(\mathrm{P}_{\mathrm{i}}\right)$ & 0,7747 & $-0,5894$ \\
Varianza de Shukla $\left(\mathrm{o}^{2}\right)$ & 0,9318 & 0,2831 \\
Ecovalencia de Wricke $\left(\mathrm{W}_{\mathrm{i}}\right)$ & 0,9320 & 0,2848 \\
Índice no paramétrico de Huhen $\left(\mathrm{S}_{\mathrm{i}}{ }^{(1)}\right)$ & 0,7869 & 0,4439 \\
Índice no paramétrico de Huhen $\left(\mathrm{S}_{\mathrm{i}}^{(2)}\right)$ & 0,8435 & 0,3967 \\
Coeficiente de regresión $\left(\mathrm{b}_{\mathrm{i}}\right)$ & $-0,6253$ & 0,6868 \\
Desviaciones de regresión $\left(\mathrm{S}^{2} \mathrm{~d}_{\mathrm{i}}\right)$ & 0,4247 & 0,5993 \\
\hline
\end{tabular}

aestivum L., Huehn 1990) y remolacha azucarera (Beta vulgaris L., Piepho y Lotito 1992), $\sigma_{\mathrm{i}}^{2}$, $\mathrm{W}_{\mathrm{i}}, \mathrm{S}_{\mathrm{i}}^{(1)}$ y $\mathrm{S}_{\mathrm{i}}^{(2)}$ clasificaron a los genotipos en el mismo orden. En maíz (Castañón y Tosquy 1996) y trigo (Rodríguez et al. 2002) se observaron resultados similares entre los índices de Eberhart y Russell (1966), de Shukla (1972) y de Wricke (1962). Piepho y Lotito (1992), Huehn (1990) y Pham y Kang (1988) detectaron que $b_{i}$ fue independiente de $\sigma_{i}^{2}, W_{i}, S_{i}^{(1)}, S_{i}^{(2)}$ y $S^{2} d_{i}$, por lo que los parámetros de Eberhart y Russell (1966), o b en cualquier combinación con $\sigma^{2}, \mathrm{~W}_{\mathrm{i}}, \mathrm{S}_{\mathrm{i}}^{(1)}, \mathrm{S}_{\mathrm{i}}^{(2)}$, producirán resultados diferentes. La no asociación lineal entre $R G$ y $S^{2} d_{i}$ que se observó en la presente investigación también fue detectada por Pham y Kang (1988). Mejía y Molina (2003) determinaron que RG y $b_{i}$ estuvieron correlacionados y ambos no tuvieron relación con $\mathrm{S}^{2} \mathrm{~d}_{\mathrm{i}}$.

El método de Eberhart y Russell (1966) es muy popular en la literatura especializada, pero ha sido muy criticado debido a la dependencia de los efectos ambientales de las variedades usadas y la no linealidad de las respuestas de éstas al medio ambiente (Shukla 1972). Sin embargo, como Márquez (1991) lo señaló, este método puede ser de gran utilidad para determinar adaptabilidad $\mathrm{y}$, por lo tanto, asociación genotipo $\mathrm{x}$ ambiente, al permitir la identificación y la recomendación de genotipos con la mayor respuesta a ambientes favorables y no favorables (estabilidad dinámica o agronómica); además, $\sigma^{2}{ }_{\mathrm{i}} \mathrm{y} \mathrm{W}_{\mathrm{i}}$ dan la contribución total de la suma de cuadrados de la IGA, por lo que ambos son menos informativos que este método. De acuerdo con Carballo y Márquez (1970), los genotipos con índices $b_{i} y$ $\mathrm{S}^{2} \mathrm{~d}_{\mathrm{i}}$ mayor que 1 e igual a 0 , respectivamente, tienen respuesta buena a ambientes favorables y son consistentes, mientras que los que tienen un índice menor a 1 e igual que 0 presentan respuesta buena a ambientes desfavorables y son consistentes. Los genotipos estables, o sea, los de mayor adaptabilidad, son los que tienen valores de 1 y 0 , respectivamente.

Los resultados anteriores sugieren también que uno de los índices no paramétricos $\left(\mathrm{S}_{i}^{(1)}\right.$ ó $\mathrm{S}_{\mathrm{i}}^{\left({ }^{(2)}\right)}$ podría emplearse en sustitución de $S_{i}, \sigma_{i}^{2}, W_{i}$ y $\mathrm{S}^{2} \mathrm{~d}_{\mathrm{i}}$. Los primeros no proporcionan información sobre adaptabilidad, pero al emplear rangos son fáciles de calcular e interpretar, no requieren de suposiciones de normalidad y homogeneidad de varianzas, en genotipos no adaptados su sesgo es pequeño, y son fáciles de calcular (Huehn 1996).

La alta correlación negativa y significativa que se observó entre $\mathrm{RG}$ y $\mathrm{P}_{\mathrm{i}}$ (Figura 1) se explica por el hecho de que el segundo se calcula a partir de suma de cuadrados de las diferencias entre el genotipo de interés con respecto al genotipo de mayor rendimiento para cada ambiente $\left(\mathrm{M}_{\mathrm{i}}\right)$, por 
lo que al incrementarse el RG las diferencias con relación a la máxima respuesta son más pequeñas; además, $\mathrm{P}_{\mathrm{i}}$ representa al cuadrado medio del efecto conjunto de genotipos e IGA y, al ser calculado con relación a $\mathrm{M}_{\mathrm{i}}$, determina la adaptabilidad de un genotipo (Lin y Binns 1988). Kang y Pham (1991) presentaron resultados similares.

Los resultados del presente estudio coincidieron parcialmente con la clasificación de índices de estabilidad realizados por otros autores, Lin et al. (1986) agruparon los índices en 3 tipos: el primero es referido a una mínima varianza entre ambientes (como $\mathrm{S}_{\mathrm{i}}^{2}$ y $\mathrm{CV}_{\mathrm{i}}$ ), el segundo considera a un genotipo como estable si su respuesta es paralela a la de la media de todos los genotipos evaluados (como $b_{i}$, con $b=1$ como estable, $\sigma_{i}^{2} y$ $\mathrm{W}_{\mathrm{i}}$ ), y el tercero, asociado al ideal de un genotipo con desviaciones de regresión pequeñas (como $S^{2} d_{i}$ ). Flores et al. (1998) clasificaron en un grupo a $\mathrm{P}_{\mathrm{i}}, \mathrm{CV}_{\mathrm{i}}$ y al rendimiento, los parámetros de Eberhart y Russell (1966) y la varianza de Shukla (1972) fueron asignados a un segundo grupo, y los índices no parámetricos de Huehn (Nassar y Hühn 1987) fueron considerados en un tercer grupo.

Del análisis de componentes principales se puede deducir que VS-46E (24), HIT-3 (18), San Lucas (23), Ixtlahuaca (20), H-40 (15) y Chalqueño (11) fueron los genotipos más estables o deseables, debido a que no presentaron interacción positiva con ninguno de los índices de estabilidad en 3 de los 4 cuadrantes del biplot (Figura 1). Los 6 métodos de estabilidad univariados también identificaron a 5 de estos 7 genotipos como deseables (Cuadro 4). Chalqueño y ETA 13 pertenecen a la raza Chalqueño, Ixtlahuaca y San Lucas a la raza Cónico y H-40, HIT-3 y VS-46E tienen germoplasma de otras, por lo que se sugiere emplearlas en un programa de mejoramiento genético para incrementar el rendimiento de grano y la estabilidad de los maíces del Valle Toluca-Atlacomulco, México.

\section{LITERATURA CITADA}

ARELlANO J.L., TUT C., MARIA A., SALINAS Y., TABOADA O.R. 2003. Maíz azul de los Valles Altos de México. I. Rendimiento de grano y caracteres agronómicos. Revista Fitotecnia Mexicana 26:101-108.

CARBAllo A., MÁRQUEZ F. 1970. Comparación de variedades de maíz de El Bajío y la Mesa Central por su rendimiento y estabilidad. Agrociencia 5:129-146.

CASTAÑÓN G., TOSQUY O.H. 1996. Análisis de la interacción genotipo-ambiente en híbridos tropicales de maíz (Zea mays L.) con diferentes métodos. Revista Fitotecnia Mexicana 19(2):141-150.

CROSSA J. 1990. Statistical analysis of multilocations trials. Advances in Agronomy 44:55-85.

EBERHART S.A., RUSSELL W.A. 1966. Stability parameters for comparing varieties. Crop Science 6:36-40.

FLORES F., MORENO T., CUBERO J.I. 1998. A camparison of univariate and multivariate methods to analyze GxE interaction. Field Crops Research 56:271-286.

FRANCIS T.R., KANNENBERG L.W. 1978. Yield stability studies in short season maize. I. A descriptive method for grouping genotypes. Canadian Journal of Plant Science 58:1029-1034.

GÁMEZ A.J., ÁVILA M.A., ÁNGELES H., DÍAZ C., RAMÍREZ H., ALEJO A., TERRÓN A. 1996. Híbridos y variedades de maíz liberados por el INIFAP hasta 1996. Publicación Especial No. 16, Toluca, México, 102 p.

GONZÁLEZ A., SAHAGÚN J., PÉREZ D.J., DOMÍNGUEZ A., SERRATO R., LANDEROS V., DORANTES E. 2006. Diversidad fenotípica del maíz Cacahuacintle en El Valle de Toluca, México. Revista Fitotecnia Mexicana 29 (3):255-261.

GONZÁlEZ A., VÁZQUEZ L.M., SAHAGÚN J., RODRÍGUEZ J.E. 2008. Diversidad fenotípica de variedades e híbridos de maíz en el Valle Toluca, Atlacomulco, México. Revista Fitotecnia Mexicana 31(01):67-76.

HERRERA B.E., CASTILlO F., SÁNCHEZ J.J., HERNÁNDEZ J.M., DELGADO A. 2002. Uso de semilla criolla y caracteres de mazorca para la selección de semilla de maíz en México. Revista Fitotecnia Mexicana 25:17-24.

HUEHN M. 1990. Nonparametric measures of phenotypic stability. Part 1: Theory. Euphytica 47:189-194.

HUEHN M. 1996. Nonparametric analysis of genotype x environment interaction by ranks, pp. 235-271. In: 
M.S. Kang y H.G. Gauch, Jr (eds.). Genotype-byenvironment interaction. CRC Press LLC, Boca Raton, New York.

KANG M.S., MILLER J.D., DARRAH L. 1987. A note on relationship between stability variance and ecovalence. Journal of Heredity 78:107.

KANG M.S., PHAM H.N. 1991. Simultaneous selection for high yielding and stable crop genotypes. Agronomy Journal 83:161-165.

LIN C.S., BINNS M.R., LEFKOVITCH L.P. 1986. Stability analysis: Where do we stand? Crop Science 26:894900.

LIN C.S., BINNS M.R. 1988. A superiority measure of cultivar performance for cultivar $\mathrm{x}$ location data. Canadian Journal of Plant Science 68:193-198.

MÁRQUEZ F. 1991. Genotecnia Vegetal, métodos, teoría, resultados. AGT editor S.A. México, pp: 177-198.

MÁRQUEZ F. 1992. La interacción genético-ambiental en genotecnia vegetal. In: Memorias del Simposio interacción genotipo-ambiente en genotencia vegetal. 26 y 27 de marzo, Sociedad Mexicana de Fitogenética. Guadalajara, Jalisco, pp: 1-27.

MARTÍNEZ A. 1988. Diseños experimentales, métodos y elementos de teoría. Editorial Trillas, México, D. F., $756 \mathrm{p}$.

MEJÍA J.A., MOLINA J.D. 2003. Cambios de estabilidad en el rendimiento de variedades tropicales de maíz. Revista Fitotecnia Mexicana 26:89-94.

NASSAR R., HÜHN M. 1987. Studies on estimation of phenotypic stability: Test of significance for non parametric measures of phenotypic stability. Biometrics 43:45-53.

NAVA F., MEJÍA J.A. 2002. Evaluación de maíces precoces e intermedios en Valles Altos Centrales de México. II. Divergencia genética. Revista Fitotecnia Mexicana 25:187-192.

NIÑO V., NICOLÁS C., PÉREZ D.J., GONZÁLEZ A. 1998. Estudio de trece híbridos y cinco variedades de maíz en tres localidades del Valle Toluca-Atlacomulco. Revista Ciencias Agrícolas Informa 12:33-43.

PHAM H.N., KANG M.S. 1988. Interrelationships among and repeatability of several stability statistics estimated from international maize trials. Crop Science 28:925-928.
PIEPHO H.P., LOTITO S. 1992. Rank correlation among parametric and nonparametric measures of phenotypic stability. Euphytica 64:221-225.

RODRÍGUEZ J.E., SAHAGÚN J., VILLASEÑOR H.E., MOLINA J.D., MARTÍNEZ A. 2002. Estabilidad de siete variedades comerciales de trigo (Triticum aestivum L.) de temporal. Revista Fitotecnia Mexicana 25(2): 43-151.

SABAGHNIA N., DEHGHANI H., SABAGHPOUR S.H. 2006. Nonparametric methods for interpreting genotype $\mathrm{x}$ environment interaction of lentil genotypes. Crop Science 46:1100-1106.

SAHAGÚN J. 1998. Evaluaciones genotípicas en series de experimentos. Germen No. 14. Sociedad Mexicana de Fitogenética, 40 p.

SÁNCHEZ J.J. 1995. El análisis biplot en clasificación. Revista Fitotecnia Mexicana 18:188-203.

SHUKLA G.K. 1972. Some statistical aspects of partitioning genotype-environmental components of variability. Heredity 29(2):237-245.

STEEL R.G.D., TORRIE J.H. 1980. Bioestadística: Principios y Procedimientos. 2a Edición. McGraw Hill. Colombia.

VELÁZQUEZ G.A., TUT C., LOTHROP J., VIRGEN J., SALINAS Y. 2005. H-40, híbrido de maíz de grano blanco para los Valles Altos de México. Folleto técnico $\mathrm{N}^{\circ}$. 21, Centro de Investigación Regional del Centro, Campo Experimental Valle de México, INIFAP, 23 p.

WRICKE G. 1962. On a method of understanding the biological diversity in field research. Z Pflanzenzuchtg 47:92-96.

WELLHAUSEN E.J., ROBERTS L.M., HERNÁNDEZ E., en colaboración con MANGELSDORF C. 1951. Razas de maíz en México: su origen, características y distribución. Folleto técnico $\mathrm{N}^{\circ}$. 5. Oficina de Estudios Especiales, Secretaría de Agricultura y Ganadería, México, D.F., 237 p. 